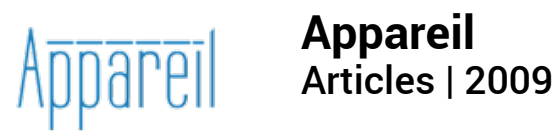

\title{
Alex invictus - Orange mécanique ou l'inservitude volontaire
}

Alain Brossat

\section{Q OpenEdition}

Journals

Édition électronique

URL : http://journals.openedition.org/appareil/698

DOI : 10.4000/appareil.698

ISSN : 2101-0714

Éditeur

MSH Paris Nord

Référence électronique

Alain Brossat, «Alex invictus - Orange mécanique ou l'inservitude volontaire », Appareil [En ligne], Articles, mis en ligne le 14 janvier 2009, consulté le 31 juillet 2020. URL : http://

journals.openedition.org/appareil/698; DOI : https://doi.org/10.4000/appareil.698

Ce document a été généré automatiquement le 31 juillet 2020.

\section{(c) (i) (9)}

Appareil est mis à disposition selon les termes de la Licence Creative Commons Attribution - Pas d'Utilisation Commerciale - Pas de Modification 4.0 International. 


\title{
Alex invictus - Orange mécanique ou l'inservitude volontaire
}

\author{
Alain Brossat
}

1 A peu près tous les films de S. Kubrick élaborent la question de la violence, et ce dans les registres les plus différents : violence de l'émancipation dans Spartacus, violence du crime dans Ultima Razzia, violences guerrières, selon des modalités bien différentes dans Les Sentiers de la gloire, Barry Lyndon et Full Metal Jacket et Dr Folamour, violence de la folie dans The Shining, violence de la passion amoureuse dans Lolita, violence de l'espèce humaine, tout simplement, dans 2001 : l'Odyssée de l'espace, etc. On pourrait aussi bien décliner cette constance dans le traitement cinématographique de la violence comme question en termes de pouvoir ou d'institution: violence de l'institution militaire, violence du pouvoir psychiatrique, violence de l'institution pénitentiaire (Orange mécanique), violence de la police, l'Etat, l'institution familiale aussi...

2 Ce qui singularise Orange mécanique (1971) dans cette constellation, c'est la confrontation de deux violences: celle, naturelle en quelque sorte, de la jeunesse indomptée, non encore saisie par les dispositifs d'apprivoisement, de domestication, et celle du processus même de civilisation activé ici par un certain nombre d'appareils ou de pouvoirs - la police, la prison, les travailleurs sociaux, les partis politiques... Ces deux violences sont symétriques en ce sens qu'elles sont portées aux extrêmes : la violence juvénile dont Alex et sa bande de "drougs" sont les vecteurs est nommée ultraviolence, dans le film comme dans le roman d'Anthony Burgess - A Clockwork Orange (1962) dont il s'inspire. La violence de l'autorité, de l'Etat, des pouvoirs et des institutions est aussi une violence extrême: au sens où elle se déploie, elle, sur le versant totalitaire, puisque son propre est de priver l'individu de sa liberté, de toute liberté en le soumettant à des formes de domestication et de conditionnement de ses comportement qui font de lui une mécanique, le séparent de son désir, le privent de toute autonomie.

3 Le modèle qui prévaut, dans le déploiement de cette violence de l'Etat destinée à domestiquer les individus et à les arracher à leur sauvagerie native, première, est un modèle médical, celui d'une médecine policière. La violence d'Alex est considéré 
comme une maladie et, dans le cours du film, on voit comment les partisans du soin correctif, qui passe par un reconditionnement de l'individu (de ses réflexes et de ses affects) l'emportent sur ceux de la sanction punitive. Alex va donc échapper à l'enfermement punitif pour être "soigné" en milieu ouvert par des méthodes "scientifiques" d'inspiration pavloviennes (le réflexe conditionné) renforcées par une pharmacopée appropriée. L'enjeu de l'extrême est ici saillant, puisque Kubrick reprend ici à son compte l'impeccable démonstration effectuée par Burgess : ce qui se présente et se légitime comme «traitement humain " paré des prestiges de la sciences et équipé d'un alibi humaniste (extraire Alex hors du monde carcéral, le réintégrer dans la société, le "guérir") s'avère, au bout du compte, confiner dangereusement avec la torture et reposer sur des techniques de conditionnement qui rappellent fâcheusement celles des régimes totalitaires... Alex, aux mains de médecins cyniques, est un cobaye, un pur objet d'expérimentation. La notion de la "santé mentale" à laquelle font référence ces médecins est fondée sur une conception purement policière de la conformité des conduites et de l'ordre social.

4 L'apologue qui semble ici suggéré par Kubrick - qui, ici, diffère sensiblement de celui qui se dégage du roman de Burgess - serait le suivant: le procès de la civilisation destiné à "guérir" les individus de leur violence native et à les rendre aptes à vivre en société est un "remède" pire que le mal. La "maladie" première des individus (leur agitation sauvage, productrice de chaos et de destructions, leur "anarchisme" spontané ou bien leur insociabilité naturelle, leur rétivité aux institutions et pouvoirs - Alex et l'école, Alex et les flics...), tout ceci est "traité", soigné et "guéri" sur un mode tel que l'individu "civilisé", c'est-à-dire domestiqué, soit réduit à une condition quasi-animale. C'est Alex qui, après avoir subi le traitement censé le rendre allergique à toute violence, se fait, chez Burgess, cette réflexion : «Am I just like some animal or dog (...) Am I just to be like a clock-work orange?»

5 Dans la fable racontée par Burgess, il apparaît qu'au bout du compte, ce processus de civilisation de l'individu, avec toute sa violence, lui laisse néanmoins une possibilité de retomber, in fine, sur ses pieds : il y a un avenir possible au delà de ces épreuves de déconditionnement et reconditionnement, et c'est cet avenir qu'évoque le dernier chapitre du livre que Kubrick ne connaissait pas lorsqu'il a élaboré son scénario (il ne figure pas dans l'édition américaine du livre sur laquelle il a travaillé), un avenir équivoque mais ouvert: Alex, dans ce dernier chapitre, entrevoit la possibilité de se "ranger", de convoler, d'avoir des enfants. Tout se passe comme s'il avait, conformément au schéma kantien, introjecté les normes et les valeurs qui supportent la sociabilité et la moralité commune, au point d'en faire les siennes propres et d'agencer sa liberté sur elle; il ne va pas nécessairement devenir un pur et simple homme de la masse, un conformiste, il va plutôt, selon cette fable, se donner à luimême un destin social conforme à son désir propre, une fois les fièvres de l'adolescence apaisées, un destin personnel agencé sur le destin collectif...

6 Mais chez Kubrick, il en va tout autrement: Alex s'en tire, in fine, en sauvant par ruse son propre désir face aux exigences exorbitantes du procès de civilisation. Sauver son désir est ici égal à sauver son goût naturel pour la violence, c'est sauver sa réserve propre de violence, tout en apprenant à la réorienter, à entrer dans la comédie humaine. Donc, au fond, il ne cède sur rien, il demeure en rupture avec le procès de la civilisation, il demeure indompté, il n'est pas devenu un esclave ou un animal, simplement, il a appris la règle $\mathrm{du}$ jeu - une règle selon laquelle seuls s'en tirent les 
cyniques, les expédients, les stratèges clairvoyants et ceux qui savent être du côté du manche. A la fin du film, il demeure un anarchiste, mais un anarchiste qui a perdu sa naïveté. Il évitera désormais les confrontations sans issue avec les institutions, et sa liberté anarchique, son goût pour la violence, il les cultivera désormais dans des jeux de connivence plus ou moins nihilistes avec les maîtres du monde. Il finira probablement vieil anar de droite, comme tant d'autres.

7 La différence entre la fable montée par Burgess et celle que propose Kubrick est donc que la première s'énonce aux conditions d'une dialectique, assez classique, celle du roman de formation: les années ou mois d'apprentissage que décrit le roman ont été terribles - mais pas vains : il y a une issue, on est dans un domaine d'expérience, pas de pure épreuve. Alex, au terme de ce processus, est mûr pour tomber amoureux, pour fonder une famille, pour vivre avec ses semblables... Alors qu'au contraire, la version kubrickienne, elle, est plutôt de facture nietzschéenne: c'est une impitoyable déconstruction du processus de la civilisation, dont la seule "leçon" qui pourrait s'en tirer serait que la seule issue pour un sujet rétif à son devenir équivalent à un animal domestique, est de préserver à tout prix et par tous les moyens sa force vitale, c'est-àdire sa réserve sauvage d'irrégulier, d'indompté, de dissident - au moins intérieur. $\mathrm{Ne}$ pas oublier la date du film: 1971 - on est dans les années incandescentes, l'attaque contre la psychiatrie policière, contre le système pénitentiaire, la vision désenchantée des élites politiques et intellectuelles portent la marque de l'irrespect généralisé qui s'étendait sur ces années-là. Il n'est pas sûr que l'on puisse qualifier cette "leçon" - qui consiste à nous dire qu'au fond l'Etat qui protège et qui civilise, les savants qui élaborent les savoirs nouveaux, les humanistes qui humanisent (etc.) sont pires que la première petite brute (blonde, en l'occurrence) venue - de cynique. Ou alors au sens du cynisme antique, un cynisme diogénique qui s'assigne la tâche première de nous ouvrir les yeux sur le jeu des puissants, de déchirer le drapé de la vie civilisée pour nous en montrer les poulies et les ficelles. Et surtout, qui, face au mensonge constitutif de la civilisation, de la culture, adopte le parti d'en rire plutôt que de succomber aux facilités d'une interminable déploration.

C'est ici que nous rejoignons directement notre sujet.

Kubrick est, en revanche, totalement fidèle à Burgess quant au ton de la narration, lequel est lui-même tout entier calqué sur ce qui constitue la grande invention du roman anglais du XVIII ${ }^{\text {ème }}$ siècle, le ton d'une narration d'une ironie plus ou moins bienveillante ou mordante, mais en tout cas destinée à établir une distance critique, une distance propice à la réflexion, entre le "héros" ou, plus généralement, les personnages et le lecteur. Fielding, Thackeray - et dont le modèle, pour moi, est le roman de Thackeray - Les Aventures de Barry Lyndon, où l'on retrouve Kubrick, ce n'est pas un hasard, tous les films de Kubrick entretenant entre eux des correspondances plus ou moins distinctes ou secrètes, mais qui assurent la solidité de l'œuvre... Ici, l'originalité du procédé mis en place par Burgess et repris par Kubrick est celle qui consiste à faire d'Alex le propre narrateur de ses propres faits et gestes, heurs et malheurs, mais dans une langue et sur un ton qui établissent une disjonction permanente avec ces faits et gestes, créent un effet de distanciation, et, au bout du compte, suscitent le rire ou bien quelque chose comme un sentiment de jubilation.

10 La narration repose en effet sur une série de procédés visant à casser l'identification du spectateur aux images violentes, aux scènes violentes, à en désamorcer la séduction : ton sentimental du roman à la Richardson (le mélo - que l'on retrouve dans la scène du 
retour de l'enfant prodigue) totalement out of time, langue du XVIII ${ }^{\text {ème }}$, adresse au spectateur (au lecteur), et aussi, bien sûr, la grande trouvaille de Burgess, recours à cet espéranto d'anticipation mêlant des mots de russe à de l'argot anglais, ceci destiné à créer un effet de trouble chronotopique en suscitant une impression de présent décalé, légèrement déplacé vers un avenir unheimlich, mais sans tomber dans le registre du roman d'anticipation. Enfin, dernier élément de narration destiné à contrarier la fascination exercée par l'ultraviolence et à déplacer celle-ci du côté d'une sorte d'excès bouffon ou de carnaval - la musique, la "grande musique" maltraitée, mutilée, dénaturée, torturée à l'électricité du synthétiseur. Ici, Kubrick dénature la musique classique pour les fins de sa narration sarcastique en pensant sans doute à ce que les nazis lui ont fait subir, en matière de détournement - qu'est-ce qu'on ne fait pas faire à la "grande" musique! Une profanation ironique.

11 Et donc, ce serait un contresens massif sur le film que d'y voir un usage opportuniste de l'ultraviolence - et de son antagonique, la violence totalitaire, celle de l'Etat et de ses institutions. Ce serait ignorer l'usage systématique de l'excès, de la surcharge, de la parodie, de la surenchère - la part déterminante du sarcasme. Le côté opérette gore, particulièrement saillant dans certaines scènes - la bagarre des drougs contre la bande de Billy Boy, notamment -. Toutes les scènes d'ultraviolence sont en quelque sorte des chorégraphies, avec un rythme trop parfait, des images trop fortes, qui sont celles de la caricature même. Tout est surjoué - ce n'est pas pour rien que Kubrick a choisi pour le rôle-titre Malcolm Mc Dowell, qui est une sorte de clown, tout comme Nicholson dans The Shining. On ne peut pas faire à Kubrick le reproche de tomber dans le panneau de l'« esthétisation de la violence ", puisqu'au contraire, il est en quête, avec ce film, d'une solution esthétique au défi que constitue le traitement au cinéma (donc en images/sons et par les moyens du montage) la présentation de formes de violence extrêmes, de conduites ultraviolentes, d'actions extrêmement violentes, y compris des actions comme l'homicide (involontaire) ou le viol sur un mode qui ne les magnifie pas, mais les expose comme problème. Alex n'est à aucun moment présenté dans le film comme un "héros", et ses drougs sont de sinistres imbéciles, comme le montre leur destin ultérieur. Leur violence n'est pas héroïsée, mais plutôt exposée comme un mirage, un faux enchantement, un misérable mirage, une drogue - une extase éphémère, suivie d'une chute sans fin. Un domaine d'intensités superficielles et fallacieuses. Ici, d'ailleurs, le premier à se tromper est Burgess qui trouve que le "passage à l'image " des scènes de violence décrites dans son film en dénature le sens et suscite une fascination suspecte pour cette violence. Mais on comprend bien pourquoi Burgess se trompe - il est un homme du livre, et il ne s'oriente pas très bien dans le monde des images.

12 Kubrick récuse également toute approche sociologique de l'ultraviolence, que ce soit celle d'une sociologie policière qui ferait d'Alex un primo-délinquant puis un récidiviste, ou bien celle d'une sociologie humaniste qui verrait en lui une victime de la société, du chômage, de la démission de l'autorité parentale et d'un urbanisme déshumanisé. Le film ne travaille aucunement dans ce type de catégorie sociologique, Alex est, comme personnage de l'ultraviolence, beaucoup plus proche des gouapes des romans de Genet (Querelle de Brest, Pompes funèbres...), des films de Pasolini (Accatone) que des « casseurs des cités » dont nous parle à longueur de pages la sociologie de bazar des journaux. Il est un curieux mélange d'insouciance sauvage, polissonne, prédatrice et de satanisme baudelairien. Dans son ultraviolence, entre en composition non 
seulement sa rétivité à la domestication, à la normation sociale, mais aussi un solide attrait pour le mal. C'est son côté non seulement indompté, mais pervers aussi. C'est sans doute ce qui a plu à Kubrick, dans le personnage imaginé par Burgess : qu'il vient nous rappeler qu'il n'est pas de vraie grande passion pour la vie, de vraie liberté sauvage sans un doigt d'attrait pour le mal, de démesure, de méchanceté et de perversité (Nietzsche). C'est le côté "diable" d'Alex.

Mais le trait de génie de Burgess auquel, ici, Kubrick emboîte le pas, est d'avoir construit son récit non pas en donnant la parole en premier lieu aux représentants de la norme appelés à juger les écarts de conduite et les exactions commises par ce diable, mais au diable lui-même dont la disposition subjective est, de façon désarmante, celle d'un innocent, d'une sorte d'enfant joueur qui s'amuse à organiser des rixes, des intrusions violentes chez les bobos, des parties à trois avec des gamines... Là encore, la narration désoriente le spectateur et l'empêche de se laisser gagner par l'esprit de sérieux d'une perception indignée de tous ces accès de violence "gratuite" : car toute cette débauche de violence "montrée" est constamment débranchée par les interventions du narrateur, avec son ton tantôt enjoué, tantôt sentimental, tantôt larmoyant - propre en tout cas à introduire la dimension d'une sorte de second degré permanent.

L'enjeu du film de Kubrick n'est pas de séduire en exhibant la "belle violence », celle du sang bien rouge qui se met à couler, comme dit Alex (mais nous ne sommes à aucun moment appelés à nous identifier à Alex), mais de construire un apologue, qui tourne autour d'une philosophie noire et dé-moralisée de la civilisation: civiliser ne consiste pas à transformer l'enfant qui est une sorte de petit animal sauvage en être moral et sociable, mais à refouler une violence avec une autre, laquelle est plutôt pire que la première. Il est bien clair que si nous ne sommes pas convoqués à nous identifier à Alex, le dispositif narratif du film étant ainsi fait que nous éprouvons à son égard toute une gamme de sentiments variés, allant de l'horreur à la compassion, le coupable se transformant en victime dans le cours du film, selon un processus destiné à brouiller tous les repères identificatoires, néanmoins, s'il est un parti de l'auteur qui se manifeste dans le film, sous une forme plus ou moins cryptée, ce serait plutôt en faveur d'Alex que de l'autorité, à travers ses différents représentants. Bien sûr, politiquement, l'alliance qui s'annonce à la fin entre le représentant de l'autorité politique («The Minister of the Interior or Inferior ", dans la version déjà très sarcastique de Burgess) dessine bel et bien les contours d'une espèce de fascisme. C'est une fin d'un "immoralisme" absolu, mais seulement pour autant qu'elle vise à nous montrer une vérité cachée de la politique contemporaine et, au delà, du processus d'apprivoisement des passions sauvages dans nos sociétés. Mais, avec tout cela, Alex demeure celui qui conserve en lui quelque chose comme une part de l'indompté, de l'ingouvernable. Et il semblerait bien que si quelque chose se garde, tous comptes faits, in extremis, d'une qualité proprement humaine, de la liberté, de la capacité d'être singulier, quelque chose qui résiste à la normation homogénéisante, c'est bien, malgré tout, Alex qui l'incarne, avec ses gros fantasmes de film X, avec la sauvagerie vraiment primaire, dans tous les sens (sans élaboration) de son désir... Et c'est la raison pour laquelle le film peut s'achever sur un formidable éclat de rire plutôt que sur une mise en garde solennelle contre les dangers du nouveau totalitarisme, des nouveaux fascismes, etc. C'est pour cela que le film de Kubrick se présente ici comme l'antagonique même du 
1984 d'Orwell, un livre non seulement médiocre, au plan littéraire, mais vraiment pénible voire affligeant par l'esprit de sérieux et le sérieux d'église qui le mine.

Le Orange mécanique de Kubrick est, à ce titre, tout sauf une de ces anti-utopies destinées à avertir les vivants sur le ton de solennité requis, des dangers mortels qui menacent leur intégrité s'ils laissent proliférer les dangers que recèlent les pouvoirs modernes; c'est au contraire une démolition joyeuse et même jubilante du récit d'édification (dans les deux sens du terme) de la modernité. Le fait que, fondamentalement et en dépit de tout, le conducteur du récit soit le rire, même dans les scènes les plus éprouvantes (le viol et l'homicide involontaire, le reconditionnement d'Alex, au cinéma...) est, fondamentalement une position philosophique. C'est un film vitaliste qui aimerait nous convaincre que le cercle de la domestication ne se referme jamais tout à fait, parce que la vie, comme flux, comme impulsion, comme énergie, finit toujours par trouver des brèches et des lignes de fuite. Alex c'est la vie, et la vie, comme principe vital, c'est fondamentalement immoral, en deçà ou par-delà la morale et, si nous le savons, alors nous ne perdons pas tout à fait le goût de rire, nous ne sommes pas encore passés du côté des araignées et des cancrelats (Zarathoustra). L'idée vitaliste, ici, qui rejoint Nietzsche, encore une fois, c'est que la vie ne peut pas s'affirmer contre tout ce qui conspire à l'éteindre sur un mode civilisé, tempéré. Elle a nécessairement un pacte avec la violence, et la violence extrême, car elle seule permet de rompre l'encerclement de la domestication, de l'animalisation du bétail humain. Donc, l'ultraviolence dont Alex et sa bande sont le vecteur ne peut pas être montrée, mise en scène sur un mode tout entier péjoratif, car ce mode péjoratif coïncide avec le regard que les ennemis de la vie, les promoteurs des disciplines, les vicaires de la police des conduites, portent sur elle. L'ultraviolence doit être aussi montrée dans son caractère éruptif et strident, comme ce qui rompt l'encerclement de la vie par les veilleurs de nuit de la domestication - même si elle s'avère à l'usage être davantage un piège qu'une solution.

Le rire n'est pas seulement "possible", dans cette optique, il est la seule perspective possible, celle de la résistance de la vie à la violence de cette forme (moderne, occidentale...) du processus de la civilisation. Le rire signale que l'espérance n'est pas éteinte: l'affaire n'est pas entendue, le zoo humain (Sloterdijk) n'est pas encore entièrement clôturé - c'est le sens de la fin "ouverte" du film, où se célèbre le retour triomphant des forces primaires de la vie: la grande santé d'Alex contre la santé médicale/policière promue par les fossoyeurs de la vie. La dimension sarcastique du film s'agence autour de la "double guérison" d'Alex: une première fois, guérison sinistre en forme de déchéance aux conditions des disciplines et des traitements de reconditionnement, une seconde aux conditions de sa résurrection (Zarathoustra, encore). Le film tourne le dos aux processus actuels sans cesse accentués, puisqu'il est un manifeste contre la médicalisation de la vie, contre son immunisation toujours croissante. Une image terrifiante et comique de cette tendance : Alex nourri à la cuillère, comme un bébé, le Nanny State et le re-devenir mineurs des sujets dans nos sociétés. Cette violence-là, qui se montre sur un mode inversé comme protection et prise en charge, ne peut être traitée que sur un mode de dérision. Et pourtant, elle est terrifiante : Alex, réduit à l'état de légume, à l'issue de son traitement et des malheurs afférents. De même, des personnages comme le gardien-chef de la prison, incarnation d'une autorité certes démodée, mais toujours agissante : il fait rire, précisément parce qu'il est le représentant pathétique d'une autorité en perte de vitesse (le disciplinaire/ punitif), et qu'au fond il est moins un salaud que bien d'autres dans le film (il est ici au 
même plan que le prêtre - les pouvoirs anciens) - les autres, qui portent moins à rire, ce sont les représentants des pouvoirs qui montent, Ludovico, le savant qui expérimente sur le vivant (cf. Milgram et son fameux test) et le ministre de l'Intérieur, manipulateur en chef et apôtre de la société de contrôle. Ces derniers font moins rire, parce qu'ils se sont emparés des motifs avantageux de la "réforme" et de l'humanisation des peines pour tenter de promouvoir leur politique sécuritaire et démagogique - ça ne vous rappelle rien?

Aux dispositifs de pouvoir traditionnels - police, justice, PJJ protection judiciaire de la jeunesse - dispositifs alliant surveillance et répression viennent se substituer de nouvelles alliances : représentants de la démocratie du public le regard rivé sur les sondages et l'horizon des élections à venir, "savants" (pouvoir médical) et, éventuellement élites intellectuelles. Une "modernité" du pouvoir qui, à l'usage, s'avère pire que les "archaïsmes" des formes antérieures.

Ici, on retrouve le procédé qui avait cours dans le roman de Vonnegut: là où se présente un certains seuil de saturation dans ce qui suscite l'effroi, l'horreur, le dégoût, le mépris, l'indignation, le découragement et qui, toujours, s'associe à des formes de violence extrême, apparaît ce débouché risqué, mais toujours possible du rire libérateur, que l'on pourrait appeler le rire trotz alledem (Heine, Biermann), le rire malgré tout, le rire-plutôt-qu'-en-pleurer (Tucholsky). Un rire qui peut se moduler selon toutes sortes de modalités : sarcastique, ironique, humoristique, mordant, énorme (Jarry), absurde, déchaîné... C'est par exemple sur ce mode que se construit la scène où s'effectue la démonstration de la guérison d'Alex, devenu tout à fait allergique à quelque violence que ce soit - le sexe, la baston. « Lerne lachen ohne zu weinen » (Tucholsky).

Le rire a ici une fonction critique. Il exprime cette sorte d'horreur sacrée que nous inspirent cette engeance. Alors qu'Alex, c'est différent, parce qu'il est pour nous audelà de toute critique, au sens où il est quelque chose comme "notre inconscient", l'inconscient de chacun d'entre nous...

\section{RÉSUMÉS}

Ce texte s'attache à montrer la proximité du grand art cinématographique développé par S. Kubrick dans ce film avec l'analytique des pouvoirs entreprise par Michel Foucault. Le motif de la plasticité et de la variabilité des dispositifs de pouvoir y est central, de même que le questionnement sur les normes et leur relativité. Pour Kubrick, comme pour Foucault, chaque dispositif de pouvoir est une singularité susceptible de se brancher sur d'autres, de l'influencer, de l'affaiblir ou au contraire de le renforcer. De même, la question de la violence est envisagée non pas tant en relation à des institutions qu'à des relations de pouvoir.

\section{INDEX}

Mots-clés : dispositif, hyperviolence, pouvoir(s), prison, psychiatrie, violence 